\title{
Altered explorative strategies and reactive coping style in the FSL rat model of depression
}

\author{
Salvatore Magara ${ }^{1}$, Sarah Holst ${ }^{1}$, Stina Lundberg ${ }^{2}$, Erika Roman ${ }^{2}$ and Maria Lindskog ${ }^{1 *}$ \\ ${ }^{1}$ Department of Neuroscience, Karolinska Institutet, Stockholm, Sweden, ${ }^{2}$ Department of Pharmaceutical Biosciences, \\ Uppsala University, Uppsala, Sweden
}

\section{OPEN ACCESS}

Edited by:

Riccardo Brambilla

San Raffaele Scientific Institute and

University, Italy

Reviewed by:

Fuat Balci,

Koc University, Turkey Nichola Brydges,

Cardiff University, UK

${ }^{*}$ Correspondence:

Maria Lindskog,

Department of Neuroscience, Karolinska Institutet, Retziusväg 8 ,

17177 Stockholm, Sweden mia.lindskog@ki.se

Received: 13 February 2015 Accepted: 25 March 2015

Published: 21 April 2015

Citation:

Magara S, Holst S, Lundberg S, Roman E and Lindskog M (2015) Altered explorative strategies and reactive coping style in the FSL rat model of depression.

Front. Behav. Neurosci. 9:89. doi: 10.3389/fnbeh.2015.00089
Modeling depression in animals is based on the observation of behaviors interpreted as analog to human symptoms. Typical tests used in experimental depression research are designed to evoke an either-or outcome. It is known that explorative and coping strategies are relevant for depression, however these aspects are generally not considered in animal behavioral testing. Here we investigate the Flinders Sensitive Line (FSL), a rat model of depression, compared to the Sprague-Dawley (SD) rat in three independent tests where the animals are allowed to express a more extensive behavioral repertoire. The multivariate concentric square field ${ }^{\mathrm{TM}}$ (MCSF) and the novel cage tests evoke exploratory behaviors in a novel environment and the home cage change test evokes social behaviors in the re-establishment of a social hierarchy. In the MCSF test, FSL rats exhibited less exploratory drive and more risk-assessment behavior compared to SD rats. When re-exposed to the arena, FSL, but not SD rats, increased their exploratory behavior compared to the first trial and displayed risk-assessment behavior to the same extent as SD rats. Thus, the behavior of FSL rats was more similar to that of SDs when the rats were familiar with the arena. In the novel cage test FSL rats exhibited a reactive coping style, consistent with the reduced exploration observed in the MCSF. Reactive coping is associated with less aggressive behavior. Accordingly, FSL rats displayed less aggressive behavior in the home cage change test. Taken together, our data show that FSL rats express altered exploratory behavior and reactive coping style. Reduced interest is a core symptom of depression, and individuals with a reactive coping style are more vulnerable to the disease. Our results support the use of FSL rats as an animal model of depression and increase our understanding of the FSL rat beyond the behavioral dimensions targeted by the traditional depression-related tests.

Keywords: multivariate concentric square field (MCSF) test, novel cage test, home cage change test, risk assessment, risk taking, social behavior, behavioral profiling, coping style

\section{Introduction}

Animal models are necessary to understand the genetic, environmental, and neurobiological underpinnings of neuropsychiatric disorders that studies in humans cannot sufficiently control for or access. The need for new experimental tests and improved animal models have been emphasized in order to gain progress in understanding pathophysiology of complex human disorders and to develop new treatment strategies (Nestler and Hyman, 2010; Anonymous, 2011). 
Depression is a broad diagnosis based on subjective symptoms of the patients. To date, we still do not understand the underlying mechanisms of depression, nor do we have reliable biomarkers. This has lead the National Institute of Health (NIH) to propose a framework to classify mental diseases based on functional constructs: the Research Domain Criteria (RDoC) (Cuthbert and Insel, 2013). Animal tests traditionally used in domains related to depression measure a single either-or behavior in response to a given, often stressful, stimulus. For example, in the forced swim test the time spent swimming vs. staying immobile is measured as the response to acute threat in the domain of negative valence, according to the RDoC. However, the exposure to stress evokes a spectrum of behavioral responses that is not taken into account in traditional tests (Kas et al., 2007), despite the knowledge that the response to novelty and coping styles in stress handling are important factors determining resilience or vulnerability to depression (Hankin, 2012). By expanding animal research beyond the use of traditional tests, this framework will facilitate the translation of experimental work into the clinic.

The Flinders Sensitive Line (FSL) is a rat model of depression. Over the years, behavioral studies on FSL rats have supported the face and predictive validity of the model in relation to human depression using traditional rodent tests for assessment of depressive-like behavior (Overstreet and Wegener, 2013). An alternative approach is to turn toward basal drives, such as exploration, risk assessment, risk taking, and shelter seeking, which are evolutionary conserved and therefore of importance from a translational perspective (Gosling, 2001; Sih et al., 2004). These aspects can be evaluated in a more complex setting where the animal is allowed to freely express a broad range of behaviors. In the multivariate concentric square field ${ }^{\mathrm{TM}}$ (MCSF) test, the animal is introduced to an arena including open areas, enriched zones to encourage exploration, sheltered areas, elevated passages, and different light conditions. This test was developed by Meyerson et al. and has been behaviorally validated with regard to identification of risky as opposed to safe areas (Meyerson et al., 2006). The MCSF test has already been used in several studies for behavioral profiling of selectively bred rat lines (Roman et al., 2007, 2012; Roman and Colombo, 2009). Another approach taking advantage of the animals' spontaneous behavior is to score continuous behaviors in a mild aversive environment such as a novel home cage without cage mates (Marques et al., 2008). The individual behaviors including exploring and passive/avoiding actions are grouped in categories based on emotional reactivity and stress coping styles (Steimer et al., 1997; Koolhaas et al., 1999; Marques et al., 2008). By including social challenges, the individual coping style can be assessed by observing the subtle dominance behavior, for example when animals re-establish their dominance-subordination relationships after a cage change in the home cage change test. The concept of stress coping styles was developed based on two opposite physiological responses to stress (Koolhaas et al., 1999). Proactive coping responses are characterized by territory control, high aggression, and a physiological response including increased blood pressure and adrenaline release. Conversely, reactive coping responses are characterized by immobility and low aggression, accompanied with decreased blood pressure and increased plasma levels of corticosterone
(Koolhaas et al., 2007, 2011). Reactive coping style and submissive behavior are associated with depression-like behavior in animal models (Blanchard and Blanchard, 1990; Hasler et al., 2004; Nesher et al., 2013).

The aim of the present study is to achieve a more detailed and nuanced analysis of explorative strategies in a complex environment and of stress coping styles in FSL rats, which has not yet been performed. Here we show that when analyzed in the MCSF, the novel cage test and the home cage change test for social interaction, the FSL rats display an altered explorative strategy compared to SD rats and are characterized by reactive coping style.

\section{Materials and Methods}

\section{Animals and Housing}

Behavioral testing was performed on age-matched male SpragueDawley (SD) (Charles River Laboratories, Germany) and FSL rats (bred in-house, three different litters) between 12 and 14 weeks of age ( $n=12$ /group, all SD rats came from different litters). SD rats (which the FSL was derived from) were used as control in order to compare the behavior of FSL rats to a well-characterized strain rather than another selectively bred line. The animals were housed under controlled room temperature $\left(21 \pm 2{ }^{\circ} \mathrm{C}\right)$ and humidity (50\%), with a reversed 12 -h light/dark cycle (lights off from 12 p.m. to 12 a.m.). The rats were grouphoused (two rats per cage) in transparent cages (1354G Eurostandard Type IV, Tecniplast, Italy) with bedding material (Aspen wood, Tapvei, Harjumaa, Estonia) and paper towels as enrichment. Food (R34 chow, Labfor, Lantmännen, Stockholm, Sweden) and water were provided ad libitum. All animal experiments were approved by the Stockholm North Committee on Ethics on Animal Experimentation and followed the guidelines of the Swedish Legislation on Animal Experimentation (Animal Welfare Act SFS1998:56) and the European Communities Council Directive (86/609/EEC).

\section{Procedure}

The experimental outline is illustrated in Figure 1A. Animals were handled daily 5 days prior to the start of the first test. Handling consisted of individual handling, weighing, and adaptation to the transportation bucket that was used to carry the rat from the home cage to the testing room. All animals were weighed before the MCSF test and before the novel cage test. FSL rats had lower body weight than SD rats at both time points (Tables S1, S2). Reduced body weight of FSL rats is well-described (Overstreet, 1993).

The MCSF testing consisted of two trials 1 week apart. Seven to nine days after the second trial of the MCSF test, the rats were exposed to the novel cage test and a week later to the home cage change test. FSL and SD rats were alternated in order to avoid day-time and order bias: individual animals were tested in the same period of the day throughout the behavioral tests $( \pm 1 \mathrm{~h})$ and the order of testing was maintained. All testing occurred during the dark period of the light/dark cycle, at least $1.5 \mathrm{~h}$ apart from the light switch, in a room where temperature, humidity, and background noise were similar to those in the housing room. 
A

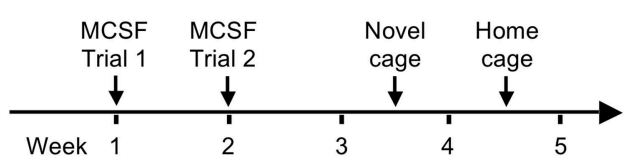

B

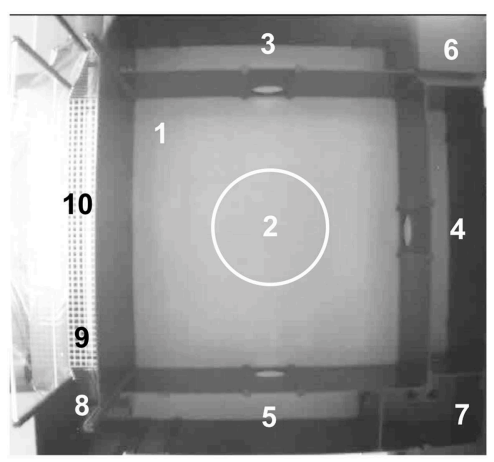

FIGURE 1 | The experimental outline (A) and the MCSF arena with the defined zones (B) numbered as follows: 1, center; 2, central circle (CTRCI); 3-5, corridors (corridors A-C); 6, dark corner room (DCR); 7, hurdle with the elevated hole board; 8, slope; 9, bridge entrance (BE); 10, bridge.

The rats were video-recorded by a digital camera placed above the test apparatus and a person blinded to the experimental groups scored the behaviors.

\section{MCSF Test}

The MCSF test was performed as previously described in detail (Roman and Colombo, 2009). The arena consists of a large square field $(100 \times 100 \mathrm{~cm})$ containing a smaller central square field $(70 \times 70 \mathrm{~cm})$. The walls are $25 \mathrm{~cm}$ high except for the walls surrounding the bridge, which are $40 \mathrm{~cm}$ high. The entire arena is divided into zones (Figure 1B): center (\#1): the square field in the middle of the arena; central circle (CTRCI, \#2): the circular area $(\emptyset=22 \mathrm{~cm})$ in the middle of the center; corridors AC (\#3-5): corridors surrounding the center; dark corner room (DCR, \#6): the covered area providing shelter; hurdle (\#7): an elevated passage with a hole board; the slope (\#8): leading to the bridge; bridge entrance ( $\mathrm{BE}, \# 9)$ : the first part of the bridge before the light source; bridge (\#10): the elevated and illuminated bridge construction. The approximate light conditions (in lux) were: center $<25$, CTRCI 30-35, corridors and hurdle $<20$, DCR $<1$ and bridge 600-850. Light intensity was measured before and after each daily session. The MCSF arena has been well-characterized to analyze exploratory behavior. Risk areas have been identified with the bridge and the central circle whereas the DCR has been shown to serve as a shelter: lactating rat dams retrieve their pups from the elevated and illuminated bridge to the DCR. Similarly, male rats collected food pellets from the bridge and hoarded and consumed the pellets in the DCR. Neither pups nor food pellets were moved out from the DCR when placed there (Meyerson et al., 2006). The areas immediately leading to the bridge (slope and bridge entrance) are used by the animal to assess the risk of visiting the bridge (risk assessment). Therefore, visits to those areas, together with the stretched attend postures, are considered as risk assessment.

The rat to be tested was transferred in a dark bucket from the home cage to the arena and released in the center facing the wall without openings. Each test session lasted $30 \mathrm{~min}$. A short representative 5-min video is attached as Supplementary Material. The arena floor and walls were wiped with $10 \%$ ethanol solution between animals and sufficient time was allowed for the floor to dry before the next rat was placed in the arena.

The number of rearing actions, stretched attend postures (SAPs), grooming actions and nose pokes into the hole board of the hurdle were scored by direct observation. Urine spots and fecal boli were counted at the end of each trial. The EthoVision system (XT 10.0, Noldus Inc., Wageningen, Netherlands) was used, where the rat was detected by using the multiple body points setting with the tail base point required for counting. Latency (LAT, s) to first visit of each zone, number (referred to as frequency, FRQ) and duration (DUR, s) of the visits, distance moved $(\mathrm{cm})$ and mean velocity $(\mathrm{cm} / \mathrm{s})$ within each zone were automatically detected. Measures for all the corridors (TOTAL CORR) and the total MCSF (ARENA) were derived. Calculated measures were: mean duration per visit to a zone (DUR/FRQ), relative frequency and duration as fraction of the total number and the total time of visits, number of rats visiting each zone or performing a particular behavior (occurrence, OCC). The descriptive parameters (Tables S1-S3) are grouped into functional categories on the basis of previous studies (Roman and Colombo, 2009; Meyerson et al., 2013). A list of abbreviations and indexes is shown in Table 1. Indexes are calculated from descriptive parameters for interpretation of impulsive-like behavior (slope/bridge interval) and anxiety-like behavior (risk/shelter indexes) as described in Table 1 (Roman et al., 2012). Here we have also introduced the shelter/corridor index as indicator of home base exploration (Table 1). Since the corridor A of the MCSF arena is the only access to the shelter, a rat that considers the shelter as a home base is more likely to enter into the DCR each time it enters the corridor A, providing us with a measurement for home base exploration. Finally, descriptive parameters within the same or similar functional category are taken into account in the trend analysis used to reveal an overall behavioral profile (Meyerson et al., 2013).

\section{Novel Cage Test}

The novel cage test was originally developed for mice to assess individual exploratory- and risk assessment-related behaviors (Marques et al., 2008). For the present study the test was adapted for rats to have a symmetric arena $(40 \times 40 \times 40 \mathrm{~cm}$, made of 
TABLE 1 | Abbreviations, indexes and trend analysis categories used in the interpretation of behaviors from the multivariate concentric square field ${ }^{\mathrm{TM}}$ (MCSF) test.

PARAMETERS
FRQ $=$ frequency (number of visits)
$\% F R Q=$ frequency as percent of the total number of visits to all zones
DUR $=$ duration (s)
DUR/FRQ = time (s) per visit
LAT = latency (s)
OCC = occurrence (the number of rats visiting each zone or
performing a particular behavior)
SAP = stretched attend posture
TOTAL ACT = number of visits to all zones
ZONES
CENTER = central field
CTRCI = central circle
CORR = corridor
DCR = dark corner room (shelter)
BE = bridge entrance

\section{INDEXES}

Shelter/corridor index $=(F R Q D C R-F R Q$ corridor $A) /(F R Q D C R+F R Q$ corridor A), measuring the recourse to the DCR on the total visits to the DCR and the corridor A. It indicates how much the shelter was considered as home base for exploration. Thus, a value close to zero indicates higher DCR base exploration.

Slope/bridge interval $=($ LAT slope - LAT bridge)/(LAT slope), indicating the delay of visiting the bridge from the slope; used as one way of interpreting impulsive-like behavior. Thus, a value close to zero indicates less risk-assessment and faster risk-taking response.

Risk/shelter duration index $=($ DUR bridge - DUR DCR $) /(D U R$ bridge + DUR DCR).

Risk/shelter frequency index $=(\mathrm{FRQ}$ bridge $-\mathrm{FRQ} \mathrm{DCR}) /(\mathrm{FRQ}$ bridge $+\mathrm{FRQ}$ $\mathrm{DCR})$, measuring the performance on the bridge compared to the DCR; used as one way of interpreting anxiety-like behavior. Thus, a higher value indicates that the animal accessed the bridge more than the DCR and is interpreted as lower anxiety-like behavior.

\section{TREND ANALYSIS}

Functional category Parameters

General activity Total act, $F R Q$ and (inv)DUR/FRQ total corridors, FRQ center, distance arena

Exploratory activity (inv)DUR total corridors, (inv)DUR center, DUR hurdle, rearings, nose pokes

Shelter seeking FRQ, DUR, and DUR/FRQ DCR

Risk assessment Stretched attend postures, DUR/FRQ slope and bridge entrance

Risk taking $\quad F R Q, D U R$, and DUR/FRQ bridge; FRQ, DUR, and DUR/FRQ central circle

The parameters indicated by (inv) were inversely ranked since they are negatively related to the functional category.

dark gray plastic) where the walls allowed the rat to perform rearings and protected from external visual cues (Steimer et al., 1997). The exploratory behaviors exhibited in the test were interpreted as indicators of emotional reactivity and coping style (Steimer et al., 1997; Koolhaas et al., 1999; Marques et al., 2008). The rat was released in the center of the arena, facing the left wall, under dimmed light (approximately 25 lux; similar to the light conditions used in the center of the MCSF arena). Each test session lasted $5 \mathrm{~min}$. The cage was cleaned with $10 \%$ ethanol solution between animals. Frequency (total number of each behavior per session) and duration (total time of each behavior per session, in seconds) of the individual behaviors described in Table 2 were manually scored with EthoLog ${ }^{\circledR}$ (Ottoni, 2000), and relative frequency and duration were calculated as fractions of the total behavior scored per rat. Individual behaviors were grouped into functional categories based on stress coping style previously described (Fernandez-Espejo and Mir, 1990; Steimer et al., 1997; Koolhaas et al., 1999) (Table 2). The scores for coping styles were calculated as the sum of relative frequency or duration of the individual behaviors grouped within the same functional category.

\section{Home Cage Change Test}

The home cage change test was performed to examine subtle dominance-subordination relationships in a home cage. Grouphoused rats were transferred into a new home cage (1354G Eurostar Type IV, Techniplast, Italy) with bedding material (Aspen wood, Tapvei, Harjumaa, Estonia) in order to simulate a cage change, and were video-recorded for $10 \mathrm{~min}$ under red light of about 3 lux. The rats were marked with a pen on their back for identification. Shuffling of bedding in front of and beside them was scored as burrowing behavior and was considered as defensive behavior (Dudek et al., 1983). Frequency (total number of each behavior per session, in bouts) and duration (total time of each behavior per session, in seconds) of the social behaviors described in Table 2 were manually scored with EthoLog ${ }^{\circledR}$ (Ottoni, 2000), and relative frequency and duration were calculated as fraction of the total social behavior scored per rat. Social behaviors were grouped into functional categories as previously described (Koolhaas et al., 1980; Fernandez-Espejo and Mir, 1990) (Table 2). The scores for the social behavior categories were calculated as the sum of relative frequency or duration of the social behaviors grouped within the same functional category.

\section{Statistical Analyses}

The majority of the behavioral data were not normally distributed according to the D'Agostino and Pearson omnibus normality test. Therefore, the statistical analysis was run by non-parametric tests. The Mann-Whitney U-test was used for comparison between experimental groups and the Wilcoxon matched pairs test was used for within-group comparison between the first and the second MCSF trial. When the rat did not enter a particular zone of the MCSF, values of dependent variables relative to that zone were considered missing, except for comparison between trials where duration and frequency measures were given as zero and latency measures were set to $1800 \mathrm{~s}$ (session duration). Intratrial time courses were analyzed with the Friedman One-Way ANOVA followed by Dunn's post-hoc test. For the indexes, an absent value was considered as missing data. The Fisher's exact test was used for analysis of occurrence. The Spearman's rank correlation test was used to check for monotonic relationships between paired data. 
TABLE 2 | Ethograms of behaviors scored in the novel cage and the home cage change tests.

\begin{tabular}{|c|c|c|}
\hline \multicolumn{3}{|l|}{ NOVEL CAGE TEST } \\
\hline Functional category & Individual behaviors & Description \\
\hline \multirow[t]{3}{*}{ Proactive coping } & Stretched approach & Walking with a flat body posture stretched and close to the floor \\
\hline & Stretched attend posture & $\begin{array}{l}\text { The rat stretches the neck or front part of the body forwards with four paws on the floor and then } \\
\text { returns backwards }\end{array}$ \\
\hline & Grooming & Scratching, shaking, wiping, or licking body parts (fur, ears, nose, and tail) \\
\hline \multirow[t]{2}{*}{ Reactive coping } & Freezing & Sudden suppression of movements \\
\hline & Motionless & Sitting or lying without suppression of movements \\
\hline \multirow[t]{2}{*}{ Exploratory activity } & Free rearing & Standing on hind legs \\
\hline & Investigating & Exploring the floor, cage walls, or air through olfactory activity \\
\hline \multirow[t]{2}{*}{ Locomotor activity } & Wall rearing & Standing on hind legs with forepaws leaning against a wall \\
\hline & Walking & Locomotor behavior with normal body posture \\
\hline \multicolumn{3}{|c|}{ HOME CAGE CHANGE TEST } \\
\hline Functional category & Social behaviors & Description \\
\hline \multirow[t]{4}{*}{ Neutral behavior } & Head-head & The head of the rat touches the head of the other rat \\
\hline & Nose-side & The rat sniffs between the ventral region and the back of the other rat \\
\hline & Nose-nose & The rat sniffs the other rat's nose in an equal sniff \\
\hline & Passing & The rat passes the other rat either in a direct meeting or from behind \\
\hline \multirow[t]{6}{*}{ Dominant behavior } & Head-tail & The head of the rat touches the tail of the other rat \\
\hline & Nose-genitals & The nose of the rat touches the genitals of the other rat \\
\hline & Following & The rat follows the other rat for more than two steps \\
\hline & Approaching & $\begin{array}{l}\text { The rat is walking or running more than three steps without hesitation with the nose pointed in direction } \\
\text { toward the other rat and fulfills the approach with a minimum of } 5 \mathrm{~cm} \text { away from the other rat }\end{array}$ \\
\hline & Nuzzling & The rat sniffs/bites/grooms the other rat in the area between the tip of the nose and the ventral region \\
\hline & Mount 1 & The rat rears and leans its front legs on the other rat's back from behind \\
\hline \multirow[t]{3}{*}{ Aggressive behavior } & Mount 2 & $\begin{array}{l}\text { The rat rears and leans its front legs on the other rat's back from behind and makes copulation } \\
\text { movements }\end{array}$ \\
\hline & Chasing & The rat runs after the other rat for more than two steps \\
\hline & Fight & Very rapid rolling, jumping, and biting of both animals while being in close contact \\
\hline \multirow[t]{3}{*}{ Submissive behavior } & Avoiding & The rat moves or faces in a direction away from the other rat when the other rat is approaching \\
\hline & Crowing under & The rat is crawling under the other rat \\
\hline & Submissive posture & The rat is lying on its back with the other rat standing and/or leaning over its ventral part \\
\hline
\end{tabular}

For analysis of behavioral profiles in the MCSF test, a trend analysis ranking procedure was used as previously described (Meyerson et al., 2013). Briefly, rats are ranked across experimental groups and trials for each parameter, and the ranking values for parameters within each functional group are summed (Table 1). Statistical analysis tested the hypothesis that experimental group or trial influences rat behavior and therefore its rank position. Since the results from the ranking were normally distributed, comparison of within-trial trend analysis was done by unpaired $t$-test, and repeated trial and interaction effect was tested by Two-Way repeated measures ANOVA with Bonferroni post-hoc analysis.

Differences were considered statistically significant at $p \leq 0.05$ and statistical trends $(T)$ were defined as $0.05<p \leq 0.1$. The
JMP 11 (SAS Institute Inc., NC, USA) and Prism 5 (GraphPad software Inc.) were used for statistical analysis.

Multivariate data analysis procedures, i.e., the Soft Independent Modeling of Class Analogy (SIMCA), were used to complement conventional statistical analyses. The principal component analysis (PCA) was use to extract and display the systemic variation in the novel cage test data from the MCSF test. The relative frequency and duration of behaviors were included in the analysis (Table S4). In the PCA, variables were pre-processed by scaling and mean-centering in order to standardize weighting of each parameter. The first component in the PCA represents the largest variation in the data set, the second component the largest of the remaining variance, etc. The PCA creates a score plot showing a summary of the relationships among the individuals, e.g., how 


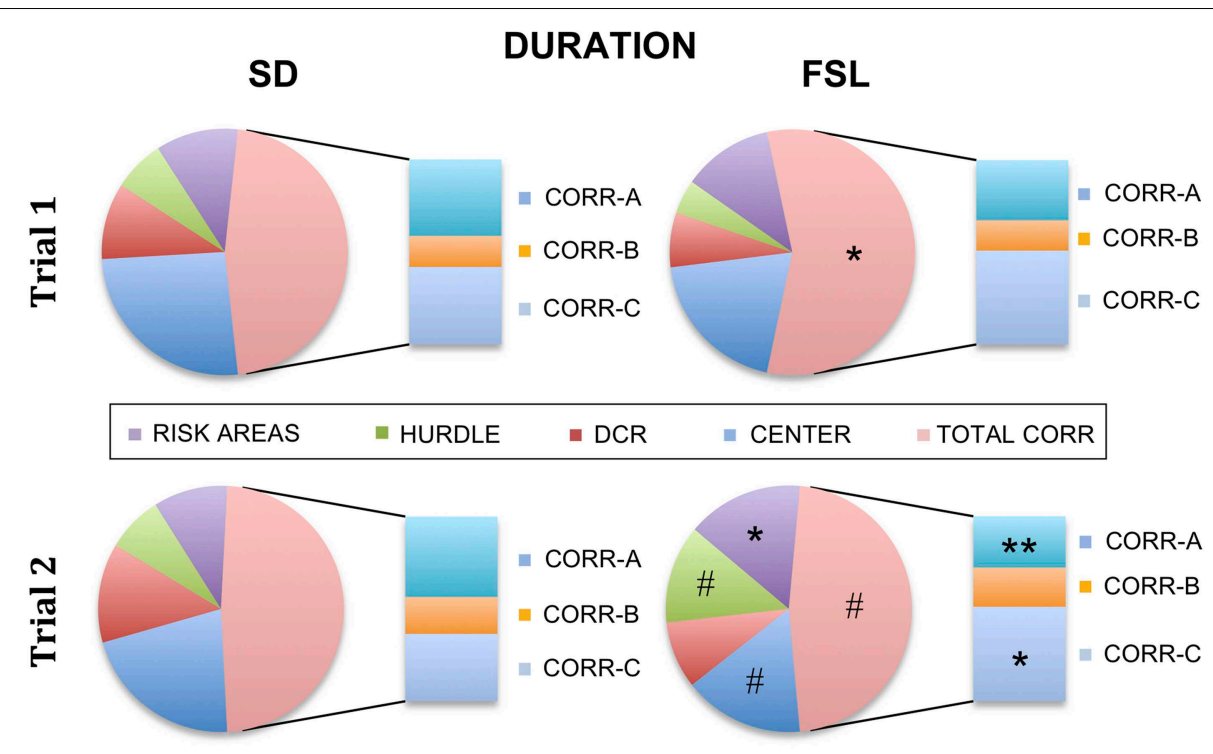

FIGURE 2 | Percent of time spent in each zone in the MCSF in relation to the total trial time for SD (left) and FSL rats (right), in trial 1 (top) and in trial 2 (bottom). The risk areas include slope, bridge entrance, bridge, and central circle. The stacked bar inserts show the time spent in the corridors $\mathrm{A}-\mathrm{C}$ as percent of the total time spent in all corridors. ${ }^{*} p<0.05,{ }^{* *} p<0.01$ Mann-Whitney U-test for comparison of FSL vs. SD rats; ${ }^{\#} p<0.05$ Wilcoxon matched pairs test for within-group comparison of trial 2 vs. trial 1 . individuals cluster in groups, and a loading plot identifying variables important for creating these relationships, i.e., behavioral parameters. The direction of the score plot corresponds to the direction in the loading plot (Jackson, 1991) (Eriksson et al., 2006).

PLS-DA is a regression extension of PCA and calculates the relationship between a Y-matrix (here experimental groups, i.e., SD and FSL rats) and an X-matrix (here MCSF parameters). The weights for the $\mathrm{X}$-variables (in the analysis denoted $\mathrm{w}$ ) indicate the importance of these variables, while the weights for the Y-variables (in the analysis denoted c) indicate which Y-variables are modeled in the respective PLS model dimensions. When these coefficients are plotted, a picture showing the relationships between $X$ and Y is obtained (Eriksson et al., 2006). The two groups will locate opposite to each other and the parameters most characteristic for the respective group will load close to that group. The PLS-DA was used for analysis of performance in the first MCSF trial. The SIMCA 13.0 software (Umetrics ${ }^{\circledR}$, Umeå, Sweden) was used.

\section{Results}

\section{The First MCSF Test (Novelty)}

The descriptive parameters from the first trial of the MCSF are displayed in Table S1 and Figure 2. In the category general activity, FSL rats covered shorter distance in the center compared to SD rats. Furthermore, FSL rats were slower than SD rats in the corridors (Table S1). In exploratory activity, FSL rats spent longer time in the corridors (Figure 2, Table S1) and less time per visit in the hurdle (Table S1) compared to SD rats. Shelterseeking associated parameters differed between the groups, where
FSL rats made fewer visits to the DCR than SD rats (Table S1). Moreover, risk-assessment behavior differed between groups, with FSL rats displaying more stretched attend postures than SD rats (Table S1). For the descriptive measures with relevance for risk-taking behavior, FSL rats differed from SD rats by a shorter latency to first visit of the central circle and a decreased velocity in the central circle (Table S1). In agreement with fewer visits to the DCR, the shelter/corridor index in FSL rats was lower than in SD rats (Figure 3A). The indexes of impulsive-like and anxiety-related behaviors (slope/bridge interval and risk/shelter indexes, respectively) did not differ between FSL and SD rats (Figures 3B-D).

The pattern of behavior is further illustrated in the PLS-DA analysis (Figure S1). The parameters that were significantly different between the groups loaded close to the group where they contributed most to the model. In addition, parameters with a statistical trend in the traditional statistical analysis (marked with a " $T$ " in Table S1) loaded close to the SD rats. Moreover, FSL rats were characterized by generally longer, although not statistically significant, latency measures relative to SD rats (Table S1) and in the PLS-DA all latencies except the latency to first visit the central circle are located on the same side as the FSL rats.

The overall behavioral profile shown by the trend analysis (Figure 4), in which the individual strategies within the same context are taken into account, revealed higher risk assessment and a trend ( $p=0.078$ ) for lower shelter-seeking behavior in FSL compared to SD rats.

Habituation to the arena, shown as the total number of visits (total act) and distance in the total arena, rearings and stretched attend postures per 10-min bins (Figure 5), was similar in FSL and SD rats. Both groups had a reduction in distance covered and 


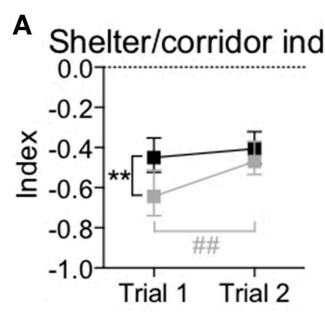

C

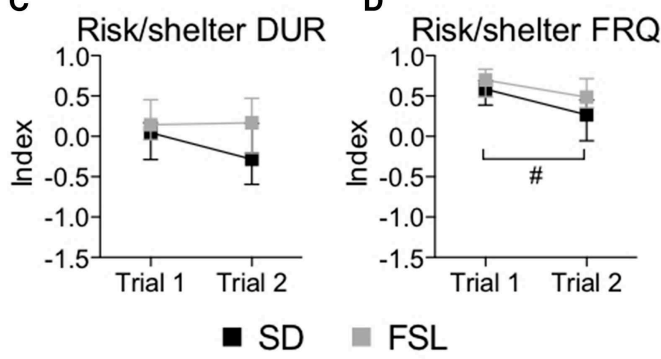

FIGURE 3 | Indexes used for interpretation of performance in trial 1 and trial 2 in the MCSF test in SD and FSL rats. Values are shown as median and interquartile range for the following indexes: shelter/corridor index used for home base behavior (A), slope/bridge interval for interpretation of impulsive-like behavior (B), and risk/shelter duration (C) and frequency (D) indexes used for interpretation of anxiety-like behavior. ${ }^{* *} p<0.01$ Mann-Whitney U-test for comparison of FSL vs. SD rats; ${ }^{\#} p<0.05$,

${ }^{\# \#} p<0.01$ Wilcoxon matched pairs test for within-group comparison of trial 2 vs. trial 1 (the black symbol \# refers to the SD group, the gray symbols \#\# to the FSL group).

stretched attend postures during the 30-min trial [distance FSL: $F_{(2,22)}=18.5, p<0.0001 ; \mathrm{SD}: F_{(2,22)}=14, p=0.0009$; SAPs FSL: $F_{(2,22)}=17.9, p<0.0001 ;$ SD: $\left.F_{(2,22)}=10.8, p=0.0044\right]$.

\section{The Repeated MCSF Test (Trial 2)}

The descriptive parameters from the second trial in the MCSF are displayed in Table S2 and Figure 2. In the general activity category, FSL rats covered shorter distance in the center than SD rats (Table S2). No differences between FSL rats and SD rats were found among descriptive parameters associated with exploratory activity (Table S2). For shelter seeking-related parameters, FSL rats displayed longer latency to reach the DCR and made fewer visits than SD rats (Table S2). Accordingly, the percent of time spent in the corridor A, i.e., the access way to the DCR, was lower in FSL compared to SD rats (Figure 2). No difference was found in the shelter/corridor index (Figure 3A). In measures associated with risk assessment and risk taking, FSL rats spent longer time than SD rats in the bridge entrance (Table S2) and in the risk areas in proportion to other zones (Figure 2). Accordingly, the percent of time spent in corridor $\mathrm{C}$ was higher in FSL rats compared to SD rats, since corridor $\mathrm{C}$ is the access way to the bridge (Figure 2). Both the slope/bridge interval and the risk/shelter indexes did not significantly differ between groups (Figures 3B-D).

The overall behavioral profile shown by the trend analysis (Figure 4) revealed no significant differences between the groups.

Time course in 10-min bins in trial 2 showed that FSL rats displayed habituation in the distance covered to the same extent as $\mathrm{SD}$ rats. The number of stretched attend postures decreased over time only in FSL rats. In SD rats, the number of visits, distance and rearings decreased over time [total act SD: $F_{(2,22)}=6.5, p=$ 0.0388 ; distance FSL, $F_{(2,22)}=12.5, p=0.0019 ; \mathrm{SD}, F_{(2,22)}=$ $13.5, p=0.0012$; rearings SD, $F_{(2,22)}=11.4, p=0.0034$; SAPs FSL, $F_{(2,22)}=9.8, p=0.0074$; Figure 5].

\section{Differences in the Familiarization to the MCSF (Trial 2 vs. Trial 1)}

The difference between a familiar (trial 2) and a novel (trial 1) environment with regard to descriptive parameters is displayed in Table S3 and Figure 2. Many of these differences are to be expected and prove that the animals recognize the arena and have established a memory from the first trial. Indeed, general activity was lower in both groups in the second compared to the first trial [trial effect $F_{(1,22)}=8.5, p=0.008$, Figure 4A]. SD rats showed a decrease in the risk/shelter index (interpreted as more anxietyrelated behavior) and an increase in the slope/bridge interval index (interpreted as higher impulsive-like behavior) in the second vs. the first trial, while no significant difference was found for FSL rats (Figures 3B-D). The index related to the home base behavior (shelter/corridor index) increased in FSL but not in $\mathrm{SD}$ rats in trial 2 compared to trial 1 (Figure 3A). Despite this increase in the use of the shelter, the trend analysis showed that FSL rats tended $(p=0.084)$ to display less shelter-seeking behavior than SD rats. The main finding was an overall increase in exploratory activity in FSL rats in the second compared to the first trial, in contrast to no change in SD rats. Indeed, the trend analysis showed a strain $\times$ trial interaction $\left[F_{(1,22)}=15.7, p=\right.$ 0.0007] and trial effect $\left[F_{(1,22)}=14.0, p=0.0011\right]$ (Figure 4B).

\section{Novel Cage Test}

The descriptive parameters from the novel cage test are displayed in Table S4. In the behaviors associated with reactive coping, both duration and frequency of motionless were higher in FSL than in SD rats. In measures related to exploratory activity, both duration and frequency of investigating behavior were lower in FSL than in SD rats (Table S4). In agreement, the comparison of the scores for coping styles revealed that frequency and duration of the reactive coping style were higher in FSL rats compared to SD rats (Figure 6). The exploratory coping duration was significantly shorter in FSL compared to SD rats. No differences between groups were found for the proactive and the locomotor coping styles (Figure 6).

The reactive coping style data were partly confirmed by the PCA (Figure S2). In the score plot, FSL rats were mostly located in the upper right and center, whereas SD rats had a more spreadout location in the lower right and upper left quadrants, but the two groups were not completely separated. The FSL group localization in the score plot (Figure S2A) corresponded to higher frequency and duration of motionless, characteristic of a reactive coping style, and stretched attend postures (Figure S2B). The investigative behavior, that was lower in the FSL rats, loaded opposite to motionless. The two principal components explained $51 \%$ of the variance $\left(R^{2} X=0.51 ; Q^{2} X=-0.02\right)$ and values of explained variation and predicted variation were within a range previously observed for biological data (Roman and Colombo, 


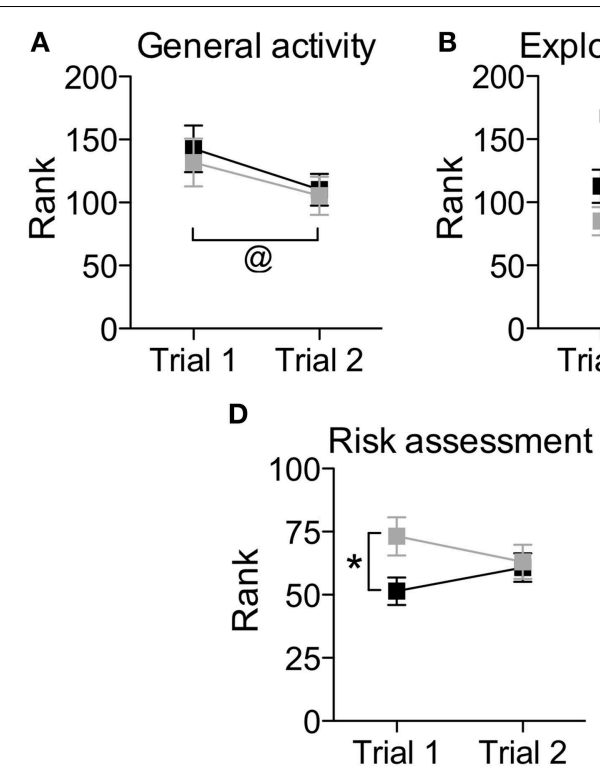

FIGURE 4 | Trend analysis of behavioral profiles in trial 1 and trial 2 in the MCSF test in SD and FSL rats. Values are shown as mean \pm SEM for the functional categories general activity (A), exploratory activity (B), shelter seeking (C), risk assessment (D), and risk taking (E). Repeated measures ANOVA was used to test for
2009; Lundstedt-Enkel et al., 2010; Meyerson et al., 2013; Palm et al., 2014).

\section{Home Cage Change Test}

In the home cage change test, there was no difference between the groups in the total score for duration and frequency of all social behaviors (data not shown). The descriptive parameters from the home cage change test are displayed in Table S5 as relative duration and frequency values. No major differences were observed in the category of neutral behaviors. In the category of dominant behaviors, both duration and frequency of the parameter mount 1 were higher in FSL than in SD rats, while the nuzzling measures were lower in FSL than in SD rats. With regard to aggressive behaviors, FSL rats exhibited less fighting than SD rats (in duration, frequency, and occurrence). Notably, in the submissive behavior category, FSL rats exhibited less submissive postures (duration, frequency, and occurrence) and more avoiding behaviors (both in duration and frequency) compared to SD rats. In the overall analysis of social behaviors, FSL rats displayed less aggressive behavior (Figure 7A) compared to SD rats, but no net differences in the category of neutral, dominant, and submissive behaviors. Besides less aggression, FSL rats also exhibited less defensive behavior burrowing (Figure 7B) compared to SD rats. Conversely, stretched attend postures are a risk-assessment behavior related to emotionality. Group difference in stretched attend postures was observed in the novel MCSF, where this behavior is possibly evoked to a larger extent than in a more simple context as the novel cage (see Discussion) (Grewal et al., 1997). We wanted to assess the relations between stretched attend postures performed in the MCSF, the defensive-related behavior burrowing and the social aggression exhibited in the home cage change test. Testing for monotonic relationship revealed the following correlations: aggression-burrowing (both duration and frequency), moderate positive correlation $(0.40 \leq|r| \leq 0.59)$, with FSL rats clustered with low aggression and low burrowing and SD rats clustered at the opposite values (Figure 7C); burrowing-stretched attend postures, strong negative correlation $(0.60 \leq|r| \leq 0.79)$, with FSL rats clustered with low burrowing and high stretched attend postures and SD rats clustered around the opposite values (Figure 7D-left); aggression-stretched attend postures: no correlation (Figure 7D-right). Thus, characteristic features of FSL rats were low aggression, burrowing, and high frequency of stretched attend postures.

\section{Discussion}

FSL rats were tested in a multivariate environment, i.e., the MCSF arena, in order to evoke a broad behavior repertoire. The descriptive parameters together with the PLS-DA indicated slightly altered explorative strategies, and the trend analysis revealed higher risk-assessment behavior in FSL relative to SD rats in the first trial. When the rats were re-exposed to the arena in a second trial, both groups displayed reduced general activity, as expected in a familiar environment. Surprisingly, FSL rats, but not SD, increased their exploratory activity and changed their home base strategy. Notably, FSL risk-assessment behavior in trial 2 did not differ from SD rats. Taken together, the behavior of FSL rats was more similar to that of SD rats when re-exposed to the arena compared to the first trial. Moreover, coping styles related to exploration of a novel environment were assessed by the novel 

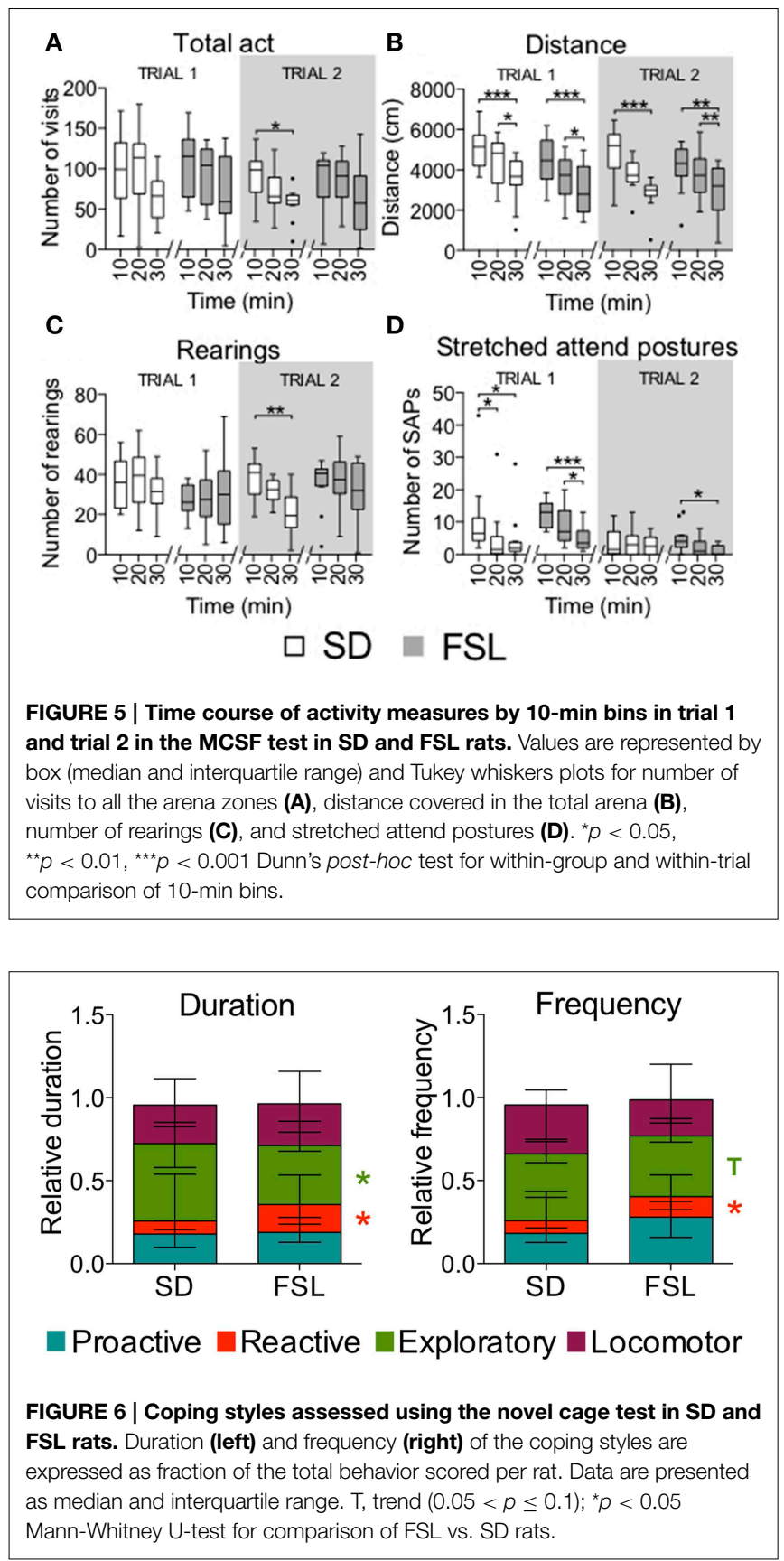

cage test and social behaviors were evaluated using the home cage change test. In good agreement with the MCSF results, FSL rats displayed more reactive coping and less exploratory behavior compared to SD rats when assessed in the novel cage test. In support of a reactive coping style, FSL rats were also characterized by less aggressive behavior in comparison with SD rats in the home cage change test.

\section{FSL Explorative Strategy in a Novel Environment}

Low novelty-induced and goal-directed exploration has been observed in FSL rats in previous studies using the open field and the novel object recognition tests, respectively (Overstreet,
1986; Gomez-Galan et al., 2013). In the novel MCSF arena, FSL rats were active and explored all zones of the arena in a fashion similar to that of SD rats. However, subtle differences between FSL and SD rats were found (Table S1). FSL rats were characterized by more time spent in the corridors and lower velocity. These results together with generally longer latency measures in FSL rats indicate a slightly lower exploratory drive compared to SD rats, in line with the previous finding that FSL rats display hypoactivity (Kokras et al., 2011). In the MCSF, the rat could choose between three corridors to leave the center and the choice of corridor affects the time for reaching the other zones. Therefore, a lack of a statistical difference in latency to the first visit of the corridors was to be expected and proved that there was no bias between the groups in choosing corridor. Exploration of a novel environment derives from the balance between risk/benefit evaluation and novelty-seeking drive (Hughes, 1997) that may regulate the use of shelter- and risk-associated areas. In the MCSF, it has been shown that the bridge is associated with risk, the DCR with shelter (Meyerson et al., 2006), and lower exploration with an increased use of the DCR (Meyerson et al., 2006; Roman et al., 2007). Surprisingly, FSL rats tended to recur less to the shelter and preferred to stay more in the corridors compared to SD rats. This was not accounted for by their reduced exploration of the arena, since the percent of visits to the DCR was still lower in FSL than in SD rats (Table S1). Rodent explorative strategies in a novel environment include the identification of a home base, i.e., a secure place where rodents often recur after each excursion into the novel territory (Eilam and Golani, 1989). The corridor A of the MCSF arena is the only access to the shelter. Therefore, the rat that considers the shelter as home base is more likely to enter into the DCR each time it enters the corridor A. The shelter/corridor index was lower in FSL compared to SD rats in the first MCSF trial (Figure 3A), suggesting that FSL rats adopted a different strategy and did not use the shelter as a home base, as SD rats did. A similar strategy has previously been described in selectively bred Sardinian alcohol-preferring rats (Roman and Colombo, 2009), however accompanied by much lower general activity, exploratory drive and risk-taking behavior than that observed in FSL rats.

\section{Altered Risk-Assessment Strategy in FSL Rats}

Risk assessment is considered a behavioral strategy to evaluate the potential risks vs. benefits deriving from exploration (Blanchard and Blanchard, 1988). A common measure of risk assessment is the stretched attend posture. This behavior has been interpreted as an indicator of anxiety-like behavior since it was affected by anxiolytic and anxiogenic drugs (Bickerdike et al., 1994; Shepherd et al., 1994). FSL rats displayed more stretched attend postures and higher general risk-assessment behavior than SD rats in the first MCSF trial (Figure 4D). In addition to the bridge, the most central part of the open center, i.e., the central circle, is considered a risk area and therefore both zones are associated with risk-taking behavior (Meyerson et al., 2006, 2013). FSL rats had a shorter latency to the first visit of the central circle, where they also moved slower than SD rats. This indicates less avoidance of the open area, in contrast to the increase in risk-assessment behavior. However, in general FSL rats performed similar to SD 

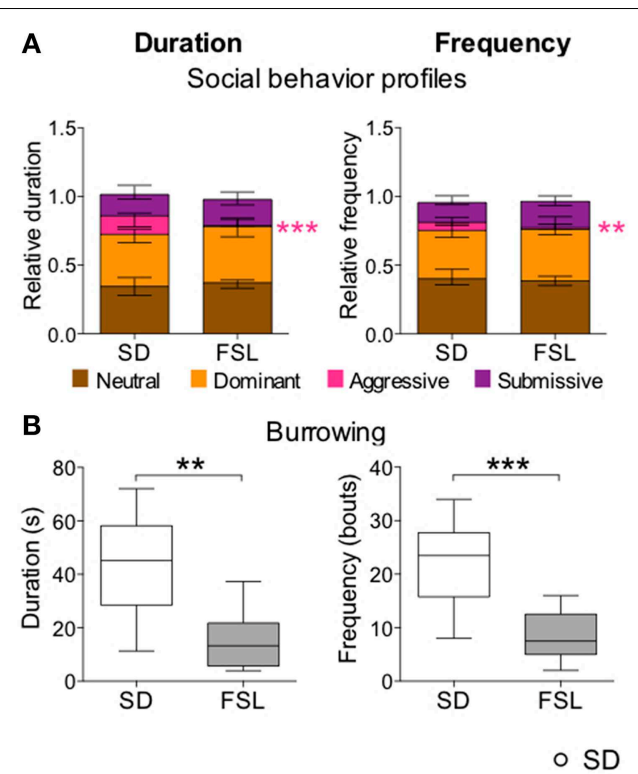

FIGURE 7 | Social behaviors (A) and burrowing (B) in the home cage change test in SD and FSL rats, and correlations between aggression and the defensive behaviors burrowing and SAPs (C,D). Duration (left) and frequency (right) of the social behavior categories (A) are expressed as fraction of the total behavior scored per rat. Data are presented as median and interquartile range. Burrowing duration (s) and frequency (bouts) (B) are represented by box (median and interquartile range) and Tukey whiskers

\section{Aggression / defensive behaviors correlations}
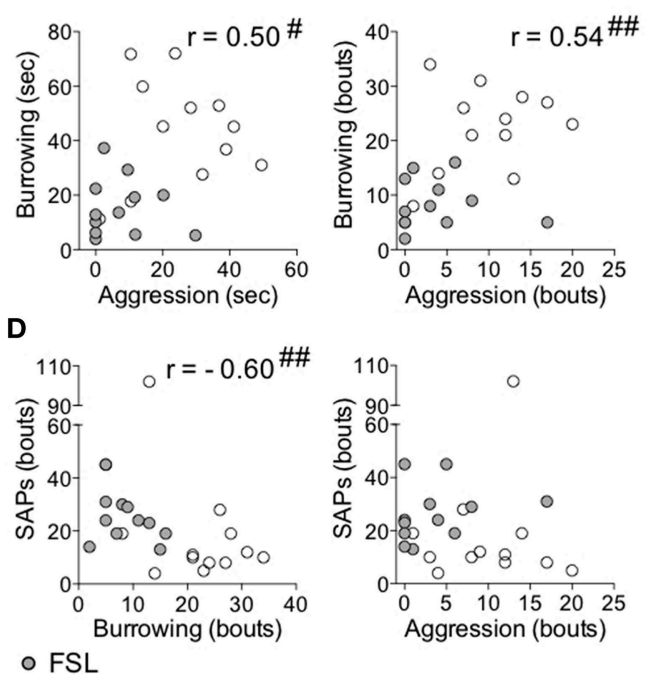

plots. Aggression, burrowing, and SAPs performed by SD and FSL rats are plotted and tested for correlations (C,D); aggression vs. burrowing duration (C-left) and frequency (C-right), burrowing vs. SAP frequency (D-left), aggression vs. SAP frequency (D-right). SAPs measures derive from the first trial of the MCSF test. ${ }^{\star \star} p<0.01,{ }^{* \star *} p<0.001$ Mann-Whitney U-test for comparison of FSL vs. SD rats; ${ }^{\#} p<0.05$, ${ }^{\#} p<0.01$ Spearman rank correlation tests, with the correlation coefficient $r$. rats in the risk areas. These observations are in agreement with previous studies where the expression of stretched attend postures did not correlate with risk-taking behavior measured as time spent in the open arm of the zero-maze test (Bickerdike et al., 1994; Shepherd et al., 1994). The fact that FSL rats did not differ from SD rats in risk-taking behavior is in agreement with the result from the risk/shelter index. This index is used for interpretation of anxiety-like behavior as it captures the performance in the risk area bridge in relation to the sheltered DCR. No difference between FSL and SD rats was found in this index (Figures 3C,D). In addition, the lower recurrence to the DCR and the efficient performance in the risk areas, supported by the overall behavioral profiling revealed by the trend analysis, indicate that FSL rats are not characterized by elevated anxiety-like behavior compared to SD rats, but by increased emotionality displayed with stretched attend postures in the MCSF and with reactive coping style in the novel cage test.

\section{Reactive Coping Style of FSL Rats is Associated with Low Aggression}

In the novel cage the challenge to explore is lower than in the MCSF, due to the absence of environmental enrichment. We suggest that this condition makes the expression of the animal behaviors more strongly driven by the animal internal stimulus or status compared to an enriched environment, and therefore it is suitable to investigate coping styles. In the novel cage test FSL rats displayed high immobility, either actively as in freezing or passively as in motionless, in agreement with unpublished separate observations in our lab. These behaviors can be summarized as reactive coping style and were higher in FSL rats compared to $\mathrm{SD}$ rats (Figure 6).

Reactive coping is also associated with low levels of aggression (Koolhaas et al., 1999). Social dominance behaviors were investigated in the home cage change test (Table S5). FSL rats exhibited less fighting and accordingly less submissive postures compared to SD rats, instead they displayed avoidance and a light version of mounting (indicated as mount 1 in Table 2). The reduced aggressive behavior displayed by the FSL rats in the home cage change test is in agreement with the reactive coping style assessed in the novel cage test. Burrowing has been described as an expression of defensive behaviors, shelter building for nesting, refuge and food storage (Bouchard and Lynch, 1989; Deacon, 2006) and can be considered as a displacement behavior for aggression when aggression is not suitable or possible to display, e.g., for a subordinate toward a dominant individual. Indeed, aggression and burrowing were positively correlated (Figure 7C), and a low expression of those behaviors characterized FSL rats. Stretched attend postures have been described as a risk-assessment strategy acted to search for and acknowledge potential threats (Blanchard and Blanchard, 1989), and related to high emotionality. In addition, when the threat is anticipated or recognized, either proactive defensive behaviors (i.e., burrowing and fighting) or reactive strategies (i.e., flight or hiding) can take place. The stretched attend postures counted during the first MCSF trial negatively correlated with the burrowing performed 
in the home cage change test (Figure 7D). Taken together, these data suggest that FSL rats display high emotionality in the potential presence of threats and the high emotionality is preferentially expressed through reactive rather than proactive responses (burrowing).

\section{Repeated Testing and Coping Style in FSL Rats}

Using repeated exposure to the MCSF arena it has previously been shown that rats remember and can recall the arena (Meyerson et al., 2006; Roman et al., 2007; Roman and Colombo, 2009). Therefore, FSL rats were tested twice in the MCSF in order to study their ability to adapt and change their behavioral strategy when re-exposed to the same context. In the second trial, FSL rats, but not SD, altered their explorative activity (Figure 4B) and home base strategy (Figure 3A) compared to the first trial. Moreover, the group differences in stretched attend postures and risk assessment that were found in the first (novel) MCSF trial were absent during the second trial (Figure 4D). These findings suggest that FSL and SD rats make a different use of the previous experience of the arena. Notably, in addition to increased exploratory activity, FSL rats spent longer time in risk areas relative to other zones compared to SD rats in the second compared to the first trial (Figure 2). This further supports a lack of anxiety-related behaviors in a multivariate environment. It has previously been demonstrated that the initial behaviors of selectively bred Alko non-alcohol rats, interpreted as anxietyrelated behaviors, became even more pronounced in a second, repeated MCSF trial exemplified by lower risk-taking behavior, shorter latency to first visit of the DCR and longer time spent in the DCR in the second trial (Roman et al., 2007). In contrast, the FSL rats do not display anxiety-like behavior in either the first or the second trial, underscoring the absence of anxietyrelated behavior. Moreover, the increase of the exploration in FSL rats in the second MCSF trial further supports a reactive coping style. Indeed, low aggression levels and a reactive coping style have been associated with flexible explorative strategies and maintained exploratory behavior in response to small context changes (Benus et al., 1991). One interpretation is that the reactive coping style of FSL rats accounts for the larger difference in MCSF performance between trials 1 and 2 compared to $\mathrm{SD}$ rats. This larger difference had as a consequence that in the familiar MCSF the behavioral profile of FSL rats was similar to that of SD rats.

\section{Risk-Assessment Strategies and Coping Styles Influence the Interpretation of Anxiety-Related Tests}

Anxiety is often, but not always, associated with depression (Hirschfeld, 2001). We have previously observed that FSL rats exhibit increased anxiety-like behaviors in a number of traditional paradigms (Femenia et al., 2015). Instead, other groups have reported that FSL rats display less (Abildgaard et al., 2011) or no (Schiller et al., 1991) anxiety-related behavior (Overstreet, 1986; Schiller et al., 1991; Overstreet et al., 2004; Abildgaard et al., 2011). Our present study suggests that FSL rats do not display increased anxiety-like behavior in the multivariate arena. It is important to consider that behavioral patterns displayed in a multivariate setting differ from those exhibited in a setting with limited number of choices (an either-or situation). For example alcohol Preferring rats have shown characteristics of increased anxiety-like behavior compared to alcohol non-preferring rats in traditional tests (Stewart et al., 1993), while the performance in the MCSF test was characterized by higher risk-taking behavior of Preferring compared to non-preferring rats (Roman et al., 2012). For the FSL rats, differences in risk-assessment strategies may be taken into account. Risk-assessment behavior is more relevantly evoked by a complex novel environment, like the MCSF arena or the canopy stretched attend posture apparatus (Grewal et al., 1997), than the open field or the novel cage test. It is possible that if the test gives the rat the opportunity to explore areas of different qualities and encourages risk assessment, such as the MCSF area (Meyerson et al., 2006, 2013), the increased risk assessment of the FSL rats allows them to perform risk-taking behavior to the same extent as the SD rats. Also the expression of coping styles may be affected. Indeed, the reactive coping style of the FSL rats (characterized by motionless and reduced investigation in the novel cage) emerges in the MCSF arena as reduced exploratory drive in the exploration of a novel environment and flexibility when familiarized to the arena, as described above, with an increase of exploratory activity in the second compared to the first trial.

\section{The FSL Rat as a Model for Depression}

Depressed patients have a reduced explorative drive for novelty, and novelty seeking inversely correlates with the severity of depressive symptoms (Hansenne et al., 1999). Likewise, proactive coping is associated with decreased risk for depression, whereas avoidance and reactive coping enhance the risk (Nagase et al., 2009; Cairns et al., 2014; Roohafza et al., 2014). However, these aspects of depression are rarely described in animal models of depression. Here we try to translate these symptoms by assessing explorative strategies in a novel complex environment, adaptation when the environment is familiar, and coping styles in the FSL model of depression. Using three independent tests based on explorative and social strategies we show that FSL rats have a reduced explorative activity that could be due to an altered strategy of exploration. This is in agreement with findings showing that rats selected for persistently low exploratory activity display increased immobility time in the forced swim, but also anxietyrelated behaviors in the elevated plus maze test (Mallo et al., 2007).

The profiling of the animals revealed that FSL rats were characterized by reactive coping style, including social dominance behaviors associated with low levels of aggression. Coping styles affect the behavioral responses to stress, testing situations, and pharmacological treatments. Indeed, selectively bred lowaggressive mice displayed reactive responses to the open field (similarly to what we observed in the FSL rats during the novel cage), increased immobility in the forced swim test and a different responsiveness to stressors and serotonergic drugs compared to high aggressive mice (Veenema et al., 2003, 2005). Our ethological observations illustrate coping-related behavioral features of FSL rats that complement our understanding of their behavior displayed in traditional depression-related tests and may be 
important to understand factors of susceptibility/resilience to stress.

\section{Conclusion}

Using a multivariate behavioral approach we demonstrated that the exploratory behavior of FSL rats is not markedly different from that of SD rats. However, subtle differences exist. In a novel environment, FSL rats are characterized by altered explorative strategies, high risk-assessment behavior and a reactive coping style compared to SD rats. When re-tested in a familiar arena, FSL rats perform similarly to SD rats, indicating the ability to familiarize with the environment, adapt and display behavioral flexibility in agreement with a reactive coping style. In addition, in the home cage change test, FSL rat strategy of social dominance behavior relies on low aggression and low defensive behaviors, but higher avoidance. Again these behaviors are associated with a reactive coping style. Our work points to the fact that coping styles affect how animals respond to experimental testing. Thus, modeling depression in animals and translating the behavioral observations between human pathology and animal behavior would benefit from using more complex

\section{References}

Abildgaard, A., Solskov, L., Volke, V., Harvey, B. H., Lund, S., and Wegener, G. (2011). A high-fat diet exacerbates depressive-like behavior in the Flinders Sensitive Line (FSL) rat, a genetic model of depression. Psychoneuroendocrinology 36, 623-633. doi: 10.1016/j.psyneuen.2010.09.004

Anonymous. (2011). Building a better mouse test. Nat. Methods 8, 697. doi: 10.1038/nmeth. 1698

Benus, R. F., Bohus, B., Koolhaas, J. M., and van Oortmerssen, G. A. (1991). Heritable variation for aggression as a reflection of individual coping strategies. Experientia 47, 1008-1019. doi: 10.1007/BF01923336

Bickerdike, M. J., Marsden, C. A., Dourish, C. T., and Fletcher, A. (1994). The influence of 5-hydroxytryptamine re-uptake blockade on CCK receptor antagonist effects in the rat elevated zero-maze. Eur. J. Pharmacol. 271, 403-411. doi: 10.1016/0014-2999(94)90800-1

Blanchard, D. C., and Blanchard, R. J. (1988). Ethoexperimental approaches to the biology of emotion. Annu. Rev. Psychol. 39, 43-68. doi: 10.1146/annurev.ps.39.020188.000355

Blanchard, D. C., and Blanchard, R. J. (1990). Behavioral correlates of chronic dominance-subordination relationships of male rats in a seminatural situation. Neurosci. Biobehav. Rev. 14, 455-462. doi: 10.1016/S0149-7634(05)80068-5

Blanchard, R. J., and Blanchard, D. C. (1989). Attack and defense in rodents as ethoexperimental models for the study of emotion. Prog. Neuropsychopharmacol. Biol. Psychiatry 13(Suppl.), S3-S14. doi: 10.1016/0278-5846(89)90105-X

Bouchard, P. R., and Lynch, C. B. (1989). Burrowing behavior in wild house mice: variation within and between populations. Behav. Genet. 19, 447-456. doi: 10.1007/BF01066170

Cairns, K. E., Yap, M. B., Pilkington, P. D., and Jorm, A. F. (2014). Risk and protective factors for depression that adolescents can modify: a systematic review and meta-analysis of longitudinal studies. J. Affect. Disord. 169, 61-75. doi: 10.1016/j.jad.2014.08.006

Cuthbert, B. N., and Insel, T. R. (2013). Toward the future of psychiatric diagnosis: the seven pillars of RDoC. BMC Med. 11:126. doi: 10.1186/1741-7015-11-126

Deacon, R. M. (2006). Burrowing in rodents: a sensitive method for detecting behavioral dysfunction. Nat. Protoc. 1, 118-121. doi: 10.1038/nprot.2006.19

Dudek, B. C., Adams, N., Boice, R., and Abbott, M. E. (1983). Genetic influences on digging behaviors in mice (Mus musculus) in laboratory and seminatural settings. J. Comp. Psychol. 97, 249-259. doi: 10.1037/0735-7036.97.3.249 test situations allowing for the expression of a broader behavioral repertoire as well as from considering coping styles of animals.

\section{Author Contributions}

ER, SH, SM, and ML designed the experiment. SM performed the animal experiments. SH, SM, and SL analyzed the data. All authors discussed the results and contributed to the writing of the paper.

\section{Acknowledgments}

Financial support from the Facias Foundation (ER), Magnus Bergvall Foundation (ML), Thuring Foundation (SH), and Vetenskapsrådet (ML) is gratefully acknowledged.

\section{Supplementary Material}

The Supplementary Material for this article can be found online at: http://journal.frontiersin.org/article/10.3389/fnbeh. 2015.00089/abstract

Eilam, D., and Golani, I. (1989). Home base behavior of rats (Rattus norvegicus) exploring a novel environment. Behav. Brain Res. 34, 199-211. doi: 10.1016/S0166-4328(89)80102-0

Eriksson, L., Johansson, E., Kettaneh-Wold, N., Trygg, J., Wikström, C., and Wold, S. (2006). Multi- and Megavariate Data Analysis. Part I: Basic Principles and Applications, 2nd Revised and Enlarged Edn., Umeå: Umetrics AB.

Femenia, T., Magara, S., DuPont, C. M., and Lindskog, M. (2015). Hippocampaldependent antidepressant action of the $\mathrm{H} 3$ receptor antagonist clobenpropit in a rat model of depression. Int. J. Neuropsychopharmacol. doi: 10.1093/ijnp/ pyv032. [Epub ahead of print].

Fernandez-Espejo, E., and Mir, D. (1990). Behavioral study in rats of paired accumbens-lesioned residents and intact intruders. Physiol. Behav. 47, 941-947. doi: 10.1016/0031-9384(90)90022-V

Gomez-Galan, M., De Bundel, D., Van Eeckhaut, A., Smolders, I., and Lindskog, M. (2013). Dysfunctional astrocytic regulation of glutamate transmission in a rat model of depression. Mol. Psychiatry 18, 582-594. doi: 10.1038/mp.2012.10

Gosling, S. (2001). From mice to men: what can we learn about personality from animal research. Psychol. Bull. 127, 45-86. doi: 10.1037/0033-2909.127.1.45

Grewal, S. S., Shepherd, J. K., Bill, D. J., Fletcher, A., and Dourish, C. T. (1997). Behavioural and pharmacological characterisation of the canopy stretched attend posture test as a model of anxiety in mice and rats. Psychopharmacology 133, 29-38. doi: 10.1007/s002130050367

Hankin, B. L. (2012). Future directions in vulnerability to depression among youth: integrating risk factors and processes across multiple levels of analysis. J. Clin. Child Adolesc. Psychol. 41, 695-718. doi: 10.1080/15374416.2012.711708

Hansenne, M., Reggers, J., Pinto, E., Kjiri, K., Ajamier, A., and Ansseau, M. (1999) Temperament and character inventory (TCI) and depression. J. Psychiatr. Res. 33, 31-36. doi: 10.1016/S0022-3956(98)00036-3

Hasler, G., Drevets, W. C., Manji, H. K., and Charney, D. S. (2004). Discovering endophenotypes for major depression. Neuropsychopharmacology 29, 1765-1781. doi: 10.1038/sj.npp.1300506

Hirschfeld, R. M. (2001). The comorbidity of major depression and anxiety disorders: recognition and management in primary care. Prim. Care Companion J. Clin. Psychiatry 3, 244-254. doi: 10.4088/PCC.v03n0609

Hughes, R. N. (1997). Intrinsic exploration in animals: motives and measurement. Behav. Processes 41, 213-226. doi: 10.1016/S0376-6357(97)00055-7

Jackson, J. E. (1991). A User's Guide to Principal Components. New York, NY: John Wiley and sons, Inc. 
Kas, M. J., Fernandes, C., Schalkwyk, L. C., and Collier, D. A. (2007). Genetics of behavioural domains across the neuropsychiatric spectrum; of mice and men. Mol. Psychiatry 12, 324-330. doi: 10.1038/sj.mp.4001979

Kokras, N., Sotiropoulos, I., Pitychoutis, P. M., Almeida, O. F., and PapadopoulouDaifoti, Z. (2011). Citalopram-mediated anxiolysis and differing neurobiological responses in both sexes of a genetic model of depression. Neuroscience 194, 62-71. doi: 10.1016/j.neuroscience.2011.07.077

Koolhaas, J. M., Bartolomucci, A., Buwalda, B., de Boer, S. F., Flugge, G., Korte, S. M., et al. (2011). Stress revisited: a critical evaluation of the stress concept. Neurosci. Biobehav. Rev. 35, 1291-1301. doi: 10.1016/j.neubiorev.2011.02.003

Koolhaas, J. M., de Boer, S. F., Buwalda, B., and van Reenen, K. (2007). Individual variation in coping with stress: a multidimensional approach of ultimate and proximate mechanisms. Brain Behav. Evol. 70, 218-226. doi: $10.1159 / 000105485$

Koolhaas, J. M., Korte, S. M., de Boer, S. F., van der Vegt, B. J., van Reenen, C. G., Hopster, H., et al. (1999). Coping styles in animals: current status in behavior and stress-physiology. Neurosci. Biobehav. Rev. 23, 925-935. doi: 10.1016/S0149-7634(99)00026-3

Koolhaas, J. M., Schuurman, T., and Wiepkema, P. R. (1980). The organization of intraspecific agonistic behaviour in the rat. Prog. Neurobiol. 15, 247-268. doi: 10.1016/0301-0082(80)90024-6

Lundstedt-Enkel, K., Bjerselius, R., Asplund, L., Nylund, K., Liu, Y., and Sodervall, M. (2010). Modeling relationships between Baltic Sea herring (Clupea harengus) biology and contaminant concentrations using multivariate data analysis. Environ. Sci. Technol. 44, 9018-9023. doi: 10.1021/es102448b

Mallo, T., Alttoa, A., Koiv, K., Tonissaar, M., Eller, M., and Harro, J. (2007). Rats with persistently low or high exploratory activity: behaviour in tests of anxiety and depression, and extracellular levels of dopamine. Behav. Brain Res. 177, 269-281. doi: 10.1016/j.bbr.2006.11.022

Marques, J. M., Olsson, I. A., Ogren, S. O., and Dahlborn, K. (2008). Evaluation of exploration and risk assessment in pre-weaning mice using the novel cage test. Physiol. Behav. 93, 139-147. doi: 10.1016/j.physbeh.2007.08.006

Meyerson, B. J., Augustsson, H., Berg, M., and Roman, E. (2006). The Concentric Square Field: a multivariate test arena for analysis of explorative strategies. Behav. Brain Res. 168, 100-113. doi: 10.1016/j.bbr.2005.10.020

Meyerson, B. J., Jurek, B., and Roman, E. (2013). A rank-order procedure applied to an ethoexperimental behavior model-the multivariate concentric square field ${ }^{\mathrm{TM}}$ (MCSF) test. J. Behav. Brain Sci. 3, 350-361. doi: 10.4236/jbbs.2013.34035

Nagase, Y., Uchiyama, M., Kaneita, Y., Li, L., Kaji, T., Takahashi, S., et al. (2009). Coping strategies and their correlates with depression in the Japanese general population. Psychiatry Res. 168, 57-66. doi: 10.1016/j.psychres.2008.03.024

Nesher, E., Gross, M., Lisson, S., Tikhonov, T., Yadid, G., and Pinhasov, A. (2013). Differential responses to distinct psychotropic agents of selectively bred dominant and submissive animals. Behav. Brain Res. 236, 225-235. doi: 10.1016/j.bbr.2012.08.040

Nestler, E. J., and Hyman, S. E. (2010). Animal models of neuropsychiatric disorders. Nat. Neurosci. 13, 1161-1169. doi: 10.1038/nn.2647

Ottoni, E. B. (2000). EthoLog 2.2: a tool for the transcription and timing of behavior observation sessions. Behav. Res. Methods Instrum. Comput. 32, 446-449. doi: 10.3758/BF03200814

Overstreet, D. H. (1986). Selective breeding for increased cholinergic function: development of a new animal model of depression. Biol. Psychiatry 21, 49-58. doi: 10.1016/0006-3223(86)90007-7

Overstreet, D. H. (1993). The Flinders sensitive line rats: a genetic animal model of depression. Neurosci. Biobehav. Rev. 17, 51-68. doi: 10.1016/S01497634(05)80230-1

Overstreet, D. H., Keeney, A., and Hogg, S. (2004). Antidepressant effects of citalopram and CRF receptor antagonist CP-154,526 in a rat model of depression. Eur. J. Pharmacol. 492, 195-201. doi: 10.1016/j.ejphar.2004.04.010
Overstreet, D. H., and Wegener, G. (2013). The Flinders sensitive line rat model of depression-25 years and still producing. Pharmacol. Rev. 65, 143-155. doi: 10.1124/pr.111.005397

Palm, S., Momeni, S., Lundberg, S., Nylander, I., and Roman, E. (2014). Riskassessment and risk-taking behavior predict potassium- and amphetamineinduced dopamine response in the dorsal striatum of rats. Front. Behav. Neurosci. 8:236. doi: 10.3389/fnbeh.2014.00236

Roman, E., and Colombo, G. (2009). Lower risk taking and exploratory behavior in alcohol-preferring sP rats than in alcohol non-preferring sNP rats in the multivariate concentric square field (MCSF) test. Behav. Brain Res. 205, 249-258. doi: 10.1016/j.bbr.2009.08.020

Roman, E., Meyerson, B. J., Hyytia, P., and Nylander, I. (2007). The multivariate concentric square field test reveals different behavioural profiles in male AA and ANA rats with regard to risk taking and environmental reactivity. Behav. Brain Res. 183, 195-205. doi: 10.1016/j.bbr.2007.06.009

Roman, E., Stewart, R. B., Bertholomey, M. L., Jensen, M. L., Colombo, G., Hyytia, P., et al. (2012). Behavioral profiling of multiple pairs of rats selectively bred for high and low alcohol intake using the MCSF test. Addict. Biol. 17, 33-46. doi: 10.1111/j.1369-1600.2011.00327.x

Roohafza, H. R., Afshar, H., Keshteli, A. H., Mohammadi, N., Feizi, A., Taslimi, M., et al. (2014). What's the role of perceived social support and coping styles in depression and anxiety? J. Res. Med. Sci. 19, 944-949.

Schiller, G. D., Daws, L. C., Overstreet, D. H., and Orbach, J. (1991). Lack of anxiety in an animal model of depression with cholinergic supersensitivity. Brain Res. Bull. 26, 433-435. doi: 10.1016/0361-9230(91) 90019-G

Shepherd, J. K., Grewal, S. S., Fletcher, A., Bill, D. J., and Dourish, C. T. (1994). Behavioural and pharmacological characterisation of the elevated "zeromaze" as an animal model of anxiety. Psychopharmacology 116, 56-64. doi: 10.1007/BF02244871

Sih, A., Bell, A. M., Johnson, J. C., and Ziemba, R. E. (2004). Behavioral syndromes: an intergrative overiew. Q. Rev. Biol. 79, 241-277. doi: 10.1086/422893

Steimer, T., Driscoll, P., and Schulz, P. E. (1997). Brain metabolism of progesterone, coping behaviour and emotional reactivity in male rats from two psychogenetically selected lines. J. Neuroendocrinol. 9, 169-175. doi: 10.1046/j.1365-2826.1997.t01-1-00571.x

Stewart, R. B., Gatto, G. J., Lumeng, L., Li, T. K., and Murphy, J. M. (1993). Comparison of alcohol-preferring (P) and nonpreferring (NP) rats on tests of anxiety and for the anxiolytic effects of ethanol. Alcohol 10, 1-10. doi: 10.1016/0741-8329(93)90046-Q

Veenema, A. H., Cremers, T. I., Jongsma, M. E., Steenbergen, P. J., De Boer, S. F., and Koolhaas, J. M. (2005). Differences in the effects of 5-HT(1A) receptor agonists on forced swimming behavior and brain 5-HT metabolism between low and high aggressive mice. Psychopharmacology 178, 151-160. doi: 10.1007/s00213-004-2005-5

Veenema, A. H., Meijer, O. C., de Kloet, E. R., and Koolhaas, J. M. (2003). Genetic selection for coping style predicts stressor susceptibility. J. Neuroendocrinol. 15, 256-267. doi: 10.1046/j.1365-2826.2003.00986.x

Conflict of Interest Statement: The authors declare that the research was conducted in the absence of any commercial or financial relationships that could be construed as a potential conflict of interest.

Copyright (c) 2015 Magara, Holst, Lundberg, Roman and Lindskog. This is an openaccess article distributed under the terms of the Creative Commons Attribution License (CC BY). The use, distribution or reproduction in other forums is permitted, provided the original author(s) or licensor are credited and that the original publication in this journal is cited, in accordance with accepted academic practice. No use, distribution or reproduction is permitted which does not comply with these terms. 